\title{
Subjective Age and Personality Development: A I0-Year Study
}

Journal of Personality 83:2, April 2015

(C) 2014 Wiley Periodicals, Inc.

DOI: 10.1 I I I/jopy. 12090

\author{
Yannick Stephan,' Angelina R. Sutin, ${ }^{2}$ and \\ Antonio Terracciano ${ }^{2}$ \\ 'University of Montpellier \\ ${ }^{2}$ Florida State University
}

\begin{abstract}
Personality theory and research typically focus on chronological age as a key indicator of personality development. This study examines whether the subjective experience of age is an alternative marker of the biomedical and psychosocial factors that contribute to individual differences in personality development. The present study uses data from the Midlife in the United States longitudinal survey $(N=3,617)$ to examine how subjective age is associated with stability and change in personality and the dynamic associations between subjective age and personality traits over a 10 -year period. Regression analyses indicated that a younger subjective age at baseline was associated with increases in Openness, Conscientiousness, and Agreeableness; correlated changes were also found. The rank-order stability of Extraversion and Openness and overall profile consistency were higher among those with a younger subjective age at baseline and were also associated with the rate of subjective aging over time. The present study reveals that beyond chronological age, the age an individual feels is related to changes in characteristic ways of thinking, feeling, and behaving over time.
\end{abstract}

In their seminal meta-analysis on the stability of personality, Roberts and DelVecchio (2000) called for alternative indicators of chronological age. They suggested that "future research on the relation between age and trait consistency may profit from assessing alternative indicators of age, such as social or psychological age" (p. 20). Indeed, empirical studies and theories of personality development have traditionally relied on chronological age as a key indicator of personality change. Yet, alternative ways of measuring developmental time may provide valuable insights into the mechanisms involved in personality development (e.g., MacDonald, DeCarlo, \& Dixon, 2011; Montepare, 2009). To that end, this study examines whether subjective age-feeling younger or older than one's chronological age - contributes to personality development over approximately 10 years. Specifically, we test whether mean-level, rank-order (at the group and individual levels), and profile stability vary by subjective age. Below, we (a) define subjective age and its association with personality and (b) describe personality development in adulthood and how subjective age may contribute to it.

\section{Subjective Age}

Age typically refers to an objective count of years since birth, but it can also be construed as a subjective phenomenon. Expressions such as "you are only as old as you feel" capture the idea that people can feel substantially younger or older than their chronological age (Kastenbaum, Derbin, Sabatini, \&
Artt, 1972). And, in fact, most adults report that they feel younger than their actual age (Gana, Alaphilippe, \& Bailly, 2004; Rubin \& Berntsen, 2006).

Adopting a younger subjective age may be one strategy individuals use to cope with the psychosocial stresses of aging (Weiss \& Lang, 2012; Westerhof \& Barrett, 2005; Westerhof, Barrett, \& Steverink, 2003). As such, feeling younger than one's years tends to be more pronounced in cultures that strongly value youthfulness and stigmatize old age, such as in the United States (Westerhof et al., 2003). Older adults may use this strategy to cope with negative and threatening conceptions of growing old that emphasize age-related losses and decline (Weiss \& Freund, 2012; Weiss \& Lang, 2012). For example, experimental studies have found that when faced with negative aging stereotypes, adults tend to distance themselves from their age group and from their chronological age, an effect that increases with advancing chronological age (Weiss \& Lang, 2012). In addition to distancing themselves from their own age group, older adults also tend to perceive themselves as more similar in age to middle-aged people when confronted with negative age-related information (Weiss \& Lang, 2012).

Correspondence concerning this article should be addressed to Yannick Stephan, EA 4556 EPSYLON, Laboratory Dynamic of Human Abilities and Health Behaviors, Department of Sport Sciences, Psychology and Medicine, University of Montpellier and St-Etienne, 4, Boulevard Henry IV, 34000 Montpellier, France. Email: yannick.stephan@univ-montpl.fr 
In addition to a coping strategy, subjective age is also a marker of physical and psychological aging (Infurna, Gerstorf, Robertson, Berg, \& Zarit, 2010; Kleinspehn-Ammerlahn, Kotter-Grühn, \& Smith, 2008; Uotinen, Rantanen, Suutama, \& Ruoppila, 2006). A younger subjective age reflects lower chronic conditions, such as hypertension and diabetes, and fewer functional limitations than an older subjective age (Demakakos, Gjonca, \& Nazroo, 2007; Infurna et al., 2010). In fact, better self-rated health explains a substantial portion of younger subjective age (Barrett, 2003; Hubley \& Russell, 2009; Infurna et al., 2010).

Subjective age has implications for a range of psychological, cognitive, and health-related outcomes across adulthood and old age. For example, feeling younger than one's chronological age predicts greater well-being (Keyes \& Westerhof, 2012; Mock \& Eibach, 2011; Stephan, Caudroit, \& Chalabaev, 2011, Westerhof \& Barrett, 2005), better perceived health (Demakakos et al., 2007; Stephan et al., 2011), and better physical and cognitive functioning (Stephan, Caudroit, Jaconelli, \& Terracciano, in press; Stephan, Chalabaev, KotterGrühn, \& Jaconelli, 2013). In contrast, feeling older than one's chronological age is a risk factor for mortality (Uotinen, Rantanen, \& Suutama, 2005). Of note, in most of these studies, subjective age rivals or outperforms chronological age as a correlate of these indicators of health and wellbeing (Demakakos et al., 2007; Kotter-Grühn, KleinspehnAmmerlahn, Gerstorf, \& Smith, 2009; Stephan, Caudroit, \& Chalabaev, 2011; Stephan et al., 2013; Westerhof \& Barrett, 2005). Taken together, subjective age is a significant alternative marker of adult development, and it may be a valuable predictor of psychological and health outcomes across the life span independent of chronological age.

From a developmental perspective, subjective age is more than a static construct. The discrepancy between subjective and chronological age emerges early in adulthood and changes across the life span (Galambos, Turner, \& Tilton-Weaver, 2005; Montepare, 2009; Rubin \& Berntsen, 2006): Younger adults feel the same age or slightly older than their actual age, but they feel increasingly younger than their actual age as they grow chronologically older (Galambos et al., 2005; Rubin \& Berntsen, 2006). The crossover between feeling older and feeling younger occurs around the age of 25 (Galambos et al., 2005; Rubin \& Berntsen, 2006). Normative development is characterized by an increasing tendency for most individuals to feel younger than their age measured in years, which translates into the maintenance of a stable relative ratio of subjective age to chronological age across adulthood and into old age (i.e., a stable proportional discrepancy; Rubin \& Berntsen, 2006; Uotinen et al., 2006). Rubin and Berntsen (2006) observed that after age 40, respondents across all age groups reported that they felt roughly $20 \%$ younger than their actual age. However, some individuals deviate from this normative trend. A reduced proportional discrepancy between subjective and chronological age over time reflects accelerated subjective aging. For example, a person who feels 40 at age 50 and then feels 55 at age 60 has experienced accelerated subjective aging. In contrast, someone who feels 40 at age 50 and then feels 45 at age 60 has experienced slower subjective aging.

Most research on personality and subjective age has only considered personality as a predictor of how young or old people feel. For example, Conscientiousness was associated with feeling younger in a clinical sample (Knoll, Rieckmann, Scholz, \& Schwarzer, 2004), whereas Extraversion and Openness have been related to a younger subjective age in community-dwelling samples (Canada, Stephan, Caudroit, \& Jaconelli, 2013; Hubley \& Hultsch, 1994, 1996; Stephan, Demulier, \& Terracciano, 2012). In a sample of adults aged 18 to 91 , this association became stronger at older ages and was independent of sociodemographic factors and health-related variables (Stephan et al., 2012). Through social comparisons of their own behaviors, thoughts, and feelings to those of their age peers and in response to personality stereotypes of aging, older adults who are extraverted and open may perceive themselves as being more similar in age to younger adults (Stephan et al., 2012). Although cross-sectional, these findings suggest that extraverted and open individuals feel increasingly younger than their age as they grow older. Longitudinal data are needed to tease apart how changes in subjective age are associated with changes in personality.

\section{Personality Development in Adulthood}

Personality development in adulthood can be conceptualized in several ways; the most commonly used metrics to track continuity and change in personality are mean-level change and rank-order stability. Mean-level change refers to changes in the average trait level of a group. Although there is some debate on the exact trajectory, cross-sectional and longitudinal studies tend to find similar normative patterns of mean-level change: Across most of adulthood, there is a decline in Extraversion, Openness, and Neuroticism and an increase in Conscientiousness and Agreeableness (Donnellan \& Lucas, 2008; Helson, Kwan, John, \& Jones, 2002; Lucas \& Donnellan, 2009, 2011; McCrae et al., 2005; Mroczek \& Spiro, 2003; Roberts, Walton, \& Viechtbauer, 2006; Small, Hertzog, Hultsch, \& Dixon, 2003; Soto, John, Gosling, \& Potter, 2011; Terracciano, McCrae, \& Costa, 2006). In addition to these normative patterns, there are also significant individual differences that lead to non-normative changes in these traits (Jackson, Thoemmes, Jonkmann, Lüdtke, \& Trautwein, 2012; Specht, Egloff, \& Schmukle, 2011; Terracciano et al., 2006; Terracciano, McCrae, \& Costa, 2010).

Accelerated subjective aging may be one factor that contributes to non-normative changes in personality over time. An older subjective age leads to the adoption of attitudes and opinions that are stereotypically associated with older people (Eibach, Mock, \& Courtney, 2010). Those who feel increasingly older over time may thus be more susceptible to stereotypes of aging and may assimilate their characteristic ways of feeling, thinking, and behaving to that of the typical older 
individual. There may also be more direct physiological pathways. There is large variability (e.g., MacDonald et al., 2011) in the physical changes associated with getting older. From the rate of skin aging to declines in energy levels and cognitive abilities, there are marked differences across individuals, and subjective age may be better than chronological age in tracking actual physiological age. For example, those who are in worse health may experience steeper declines in cognitive capacities and energy levels and thus may feel increasingly older (Kleinspehn-Ammerlahn et al., 2008; Kotter-Grühn et al., 2009). Personality traits are unlikely to be immune to such accelerated declines in body and brain functions (Robins Wahlin \& Byrne, 2011; Sutin, Costa, et al., 2013). Therefore, to the extent that accelerated subjective aging reflects accelerated physiological aging, changes in subjective age may be associated with psychological and physiologically driven changes in personality.

Rank-order stability is the degree to which the relative ordering of individuals on a given trait is maintained over time. This index of stability is commonly examined at the group level using test-retest correlations between personality scores across two points in time. The rank-order stability coefficient provides a useful description of personality stability at the group level, but it is not well suited to examine factors that contribute to individual differences in stability. Some have argued for moving from a group-level to an individual-level perspective on stability (Asendorpf, 1992; De Fruyt et al., 2006; Löckenhoff et al., 2008; McCrae, 2008; Terracciano et al., 2006, 2010). In this approach, rank-order stability is partitioned into individual contributions that provide intraindividual indices of stability (Asendorpf, 1992; Pullmann, Raudsepp, \& Allik, 2006; Terracciano et al., 2006, 2010). In addition to rank order, ipsative or profile stability tracks the intra-individual stability of configurations of traits (in contrast to individual traits), which provides information on the stability of the patterning of traits within a person across time (e.g., De Fruyt et al., 2006; Roberts, Caspi, \& Moffitt, 2001). The simultaneous assessment of profile stability across the five traits within an individual is important given that the traits coexist within the person and that they combine to contribute to one's functioning.

Personality stability is generally lower during periods of development with significant biological, cognitive, and social changes, such as adolescence, but the level of stability should be relatively constant in middle adulthood (Ardelt, 2000; Donnellan \& Robins, 2009; Lucas \& Donnellan, 2011; Lüdtke, Roberts, Trautwein, \& Nagy, 2011; Roberts \& DelVecchio, 2000; Specht et al., 2011; Terracciano et al., 2006, 2010; Trzesniewski, Donnellan, \& Robins, 2003). Still, based on the evidence that an older subjective age is associated with declining physical and cognitive conditions, we expect lower stability among individuals who feel subjectively older or who have accelerated subjective aging. Slower subjective aging may also be associated with lower stability. A younger subjective age is associated with Extraversion and Openness (Hubley \&
Hultsch, 1994; Stephan et al., 2012), and if a younger subjective age helps preserve levels of Extraversion and Openness, such individuals will also deviate from the normative trajectory of personality. Thus, personality stability may be lower for individuals at both ends of the subjective aging spectrum.

\section{The Present Research}

Using data from the Midlife in the United States longitudinal survey (MIDUS), the present study examines the longitudinal dynamics between personality and subjective age over approximately 10 years. We expect that a younger subjective age at baseline will be associated with smaller decreases in Extraversion and Openness. This hypothesis is based in part on previous cross-sectional studies (Canada et al., 2013; Hubley \& Hultsch, 1994; Stephan et al., 2012) and in part on the fact that Extraversion and Openness decline during adulthood more than other traits (Chan et al., 2012; Wood \& Roberts, 2006). We also hypothesize that a younger subjective age at baseline will be related to greater declines in Neuroticism because individuals who feel younger than their age have lower depressive symptoms, less disease burden, and better self-rated health (Keyes \& Westerhof, 2012; Kleinspehn-Ammerlahn et al., 2008; Stephan et al., 2012) and because these factors are associated with better emotional stability over time (Löckenhoff, Terracciano, \& Costa, 2009).

In addition to baseline subjective age, we hypothesize that accelerated subjective aging across the 10 years will be associated with greater declines in Extraversion and Openness than the maintenance of a stable subjective age across time. Such accelerated aging may stem from lower energy levels, declines in physical and cognitive functioning, and restricted time horizons (Kotter-Grühn et al., 2009), which could lead to declines in Extraversion and Openness. In addition, given that these changes may reflect health-related declines (Kotter-Grühn et al., 2009) that have been associated with changes in Neuroticism and Conscientiousness (Takahashi, Edmonds, Jackson, \& Roberts, 2013), we also expect that accelerated subjective aging will be associated with a decline in Conscientiousness and an increase in Neuroticism over the 10-year period.

Last, the present study also examines whether stability (rank-order, intra-individual, and profile) varies by subjective age. Given that an older subjective age is associated with declining physical and cognitive status and greater vulnerability to age stereotypes, we expect that an older subjective age at baseline will be associated with lower stability across the 10 years. Furthermore, we test for nonlinear associations between changes in subjective age and the indices of stability. Individuals who deviate from the normative trajectory of subjective age, such as those who experience accelerated or slower aging, may have lower personality stability than those who maintain a stable subjective age over the 10 -year period. 


\section{METHOD}

\section{Participants}

Data were drawn from the Midlife in the United States longitudinal survey (MIDUS I and II; Brim, Ryff, \& Kessler, 2004). The first wave of the MIDUS study collected survey data in 1994-1995 from a total of 7,108 English-speaking adults in the United States, aged 20-75 years. The baseline sample was composed of a national random digit dialing (RDD) sample $(n=3,487)$, their siblings $(n=950)$, oversamples from five metropolitan areas $(n=757)$, and a national RDD sample of twin pairs $(n=1,914)$. Participants completed a 30 -minute telephone interview and a self-administered questionnaire, which included the variables of interest of the present study. Of the 7,108 participants in MIDUS $1,4,963$ adults $(-70 \%$ of the original sample) were successfully contacted to participate in a second wave of data collection in 2004-2005. We analyzed only individuals who provided complete data for both waves on the variables of interest. The final analyzed sample had 3,617 individuals aged 24 to 75 years at baseline (55\% women; $\left.M_{\text {age }}=47.01, S D=12.30\right)$. As is typically found, those who participated in the second wave of the MIDUS showed positive selection on most variables (Radler \& Ryff, 2010). Attrition analysis revealed that the longitudinal sample had a younger subjective age, $t(6158)=-2.88, p<.01, d=0.10$, was more educated, $t(7093)=-13.73, p<.01, d=0.32$, had lower disease burden, $t(6306)=4.16, p<.01, d=0.10$, lower Neuroticism, $t(6263)=2.32, p<.05, d=0.06$, and higher Conscientiousness, $t(6268)=-6.98, p<.01, d=0.18$, than the dropout sample. Individuals in the longitudinal sample were also older, $t(7047)=4.15, p<.01, d=0.10$, and less agreeable, $t(6269)=-2.00, p<.05, d=0.04$, than those who dropped out. No significant differences were found for Extraversion and Openness.

\section{Measures}

Subjective Age. Subjective age was assessed at Wave 1 and Wave 2 by asking participants to specify, in years, how old they felt most of the time. Proportional discrepancy scores were calculated by subtracting participants' felt age from their chronological age, and these difference scores were divided by chronological age (Eibach et al., 2010; Rubin \& Berntsen, 2006; Stephan et al., 2013). A positive value indicated a youthful subjective age, and a negative value indicated an older subjective age. For example, a participant who scored +0.20 felt $20 \%$ younger, whereas a participant who scored -0.20 felt $20 \%$ older than his or her actual age. For some analyses, subjective age was divided into three groups based on the longitudinal data: an accelerated aging group, characterized by a reduced proportional discrepancy of a least one standard deviation (around 19\%) below the baseline mean of subjective age; an average aging group with a stable ratio of subjective age to chronological age within one standard deviation above and below the mean; and a slower aging group with larger proportional discrepancy of at least one standard deviation above the mean.

Personality. Personality was assessed at both waves using the Midlife Development Inventory (MIDI; Lachman \& Weaver, 1997). Participants were asked how much 25 adjectives that assessed Neuroticism, Conscientiousness, Extraversion, Openness, and Agreeableness described them on a scale ranging from 1 (not at all) to 4(a lot; for more details, see Graham \& Lachman, 2012; Turiano et al., 2012). The adjectives were moody, worrying, nervous, and calm (Neuroticism); outgoing, friendly, lively, active, and talkative (Extraversion); creative, imaginative, intelligent, curious, broad minded, sophisticated, and adventurous (Openness); organized, responsible, hardworking, and careless (Conscientiousness); and helpful, warm, caring, softhearted, and sympathetic (Agreeableness). Cronbach's alphas for each trait at Wave 1 and Wave 2, respectively, were as follows: .75 and .74 for Neuroticism, .77 and .76 for Extraversion, .77 and .77 for Openness, .56 and .58 for Conscientiousness, and .81 and .80 for Agreeableness.

Covariates. Age (in years), sex (coded as 1 for men and 0 for women), ethnicity (coded as 1 for White and 0 for other), educational level, and disease burden were included as covariates given their relation with personality change (e.g., Löckenhoff et al., 2008; Sutin, Zonderman, Ferrucci, \& Terracciano, 2013). Age squared was also included given the nonlinear changes in personality across adulthood (Lucas \& Donnellan, 2011; Specht et al., 2011; Terracciano, McCrae, Brant, \& Costa, 2005). Education was operationalized using a scale composed of 12 intervals corresponding to sequential educational milestones, ranging from 1 (no grade school) to 12 (doctoral level degree). Disease burden was measured as the sum of diseases and conditions reported by the participants on a preestablished list (Agrigoroaei \& Lachman, 2011).

\section{Data Analysis}

To test whether baseline subjective age was associated with change in personality traits, we used multiple regression to predict each personality trait at follow-up from baseline subjective age, controlling for age, age squared, sex, ethnicity, education, disease burden, and baseline personality. To test the relation between changes in subjective age and changes in personality traits, we examined partial correlations between residual change scores for both subjective age and each personality trait, controlling for the covariates.

To test whether rank-order stability differed by subjective age, test-retest Pearson correlation coefficients between baseline and follow-up scores for the five traits were computed among individuals with an older subjective age, those with a younger subjective age, and those without any discrepancy between felt and chronological age at baseline. The relation between changes in subjective age and personality stability 
was assessed using test-retest correlations for the five traits across the three subjective aging groups (i.e., accelerated, stable, and slower).

In addition to the classic test-retest correlation at the group level, we also computed stability coefficients at the individual level. This strategy is advantageous because it provides a continuous measure of stability for each individual in the sample, and thus it is possible to conduct regression analyses that account for potential confounding factors. Individual stability (IS) coefficients were computed for each individual using Asendorpf's (1992) formula, IS $=1-\left[\left(z_{1}-z_{2}\right)^{2} / 2\right]$, where $z_{1}$ and $z_{2}$ are scores for a trait standardized across the full sample at the first and second administrations. The mean IS across all respondents is equal to the retest correlation, so each IS coefficient represents the individual's contribution to overall rankorder consistency. For each individual, we also created an overall stability coefficient as the mean IS of the five traits,

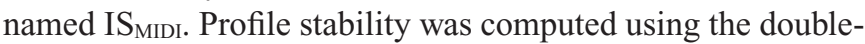
entry intra-class correlation (ICC) across the five personality traits (McCrae, 2008; Terracciano et al., 2006, 2010). Chan et al. (2012) revealed that the results obtained with the ICC are essentially the same, or even more conservative, than those obtained with other methods. Separate regression analyses were conducted with baseline subjective age as a predictor of the five IS, the IS $\mathrm{S}_{\mathrm{MIDI}}$, and the ICC as the criteria, controlling for covariates. Similarly, regression analyses were conducted to examine whether change in subjective aging over the 10 years predicted the five IS, IS $\mathrm{MIDI}$, and the ICC, controlling for the same covariates. To examine quadratic relations with the five IS, the IS $_{\mathrm{MIDI}}$, and the ICC, squared change was entered as a predictor. Due to the number of statistical tests, we took a conservative approach to significance by setting $p$ to $<.01$ (two-tailed) for all regression analyses.

\section{RESULTS}

Descriptive statistics (means and standard deviations) for all variables at Time 1 and Time 2 are presented in Table 1. An examination of mean-level change revealed that the subjective age score increased over the 10 -year period $(d=0.11)$. That is, participants felt younger than their chronological age as they grew older. In addition, all personality traits declined from baseline to follow-up $(0.08<d<0.25)$, except Conscientiousness, which was found to slightly increase $(d=0.04$; see Table 1).

\section{Subjective Age and Mean-Level Personality Change}

Partially consistent with our hypothesis, we found a positive association between baseline subjective age and change in Openness $(\beta=.06, p<.01$; see Table 2$)$. Baseline subjective age was also associated with changes in Extraversion, as hypothesized, but it did not meet our threshold for significance $(\beta=.03, p=.01)$. In contrast to our hypothesis, there was no association with Neuroticism $(\beta=-.01, p=.55$; see Table 2$)$. Unexpectedly, baseline subjective age was also positively associated with changes in Agreeableness $(\beta=.04, p<.01)$ and Conscientiousness $(\beta=.04, p<.01$; see Table 2$)$. These associations were observed both with and without demographic and health covariates in the model. Based upon the overall pattern of trait change in the MIDUS, these results suggested that those who reported a younger subjective age at baseline had a smaller decline in Openness and Agreeableness and a larger increase in Conscientiousness as compared to those who felt older.

We next examined whether change in subjective age was correlated with change in personality traits, accounting for

Table I Means, Standard Deviations, and Correlations for Study Variables at Time I and Time 2

\begin{tabular}{|c|c|c|c|c|c|}
\hline Variables & Time I & $\begin{array}{c}\text { Correlation With } \\
\text { Subjective Age Time I }\end{array}$ & Time 2 & $\begin{array}{c}\text { Correlation With } \\
\text { Subjective Age Time } 2\end{array}$ & $\begin{array}{c}\text { Correlated Change } \\
\text { Between Subjective Age } \\
\text { and Personality }\end{array}$ \\
\hline Sex (\% female) & $55 \%$ & -0.01 & & -0.01 & \\
\hline Ethnicity (\% white) & $94 \%$ & -0.01 & & -0.02 & \\
\hline Education & $7.16(2.46)$ & $0.04^{* *}$ & & $0.07 * * *$ & \\
\hline Disease burden & $2.30(2.36)$ & $-0.12 * * *$ & & $-0.08 * * *$ & \\
\hline Age & 47.01 (12.30) & $0.25^{* * *}$ & & $0.10 * * *$ & \\
\hline Subjective age & $0.15(0.19)$ & - & $0.17(0.17)^{c}$ & - & - \\
\hline Agreeableness & $3.48(0.49)$ & $0.10 * * *$ & $3.44(0.50)^{b}$ & $0.12 * * *$ & $0.06 * * *$ \\
\hline Extraversion & $3.19(0.55)$ & $0.15^{* * *}$ & $3.10(0.57)^{b}$ & $0.23 * * *$ & $0.17 * * *$ \\
\hline Neuroticism & $2.22(0.66)$ & $-0.18 * * *$ & $2.06(0.62)^{b}$ & $-0.16 * * *$ & $-0.10 * * *$ \\
\hline Conscientiousness & $3.45(0.43)$ & $0.11 * * *$ & $3.47(0.44)^{c}$ & $0.12^{* * *}$ & $0.05^{* *}$ \\
\hline Openness & $3.01(0.51)$ & $0.13^{* * *}$ & $2.90(0.53)^{b}$ & $0.21 * * *$ & $0.13^{* * *}$ \\
\hline
\end{tabular}

Note. $N=3,617$. Subjective age $=$ (chronological age-felt age)/chronological age.

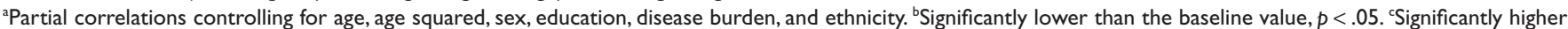
than the baseline value, $p<.05$.

$* p<.05$. ** $p<.01$. **** $p<.001$ 
Table 2 Summary of Regression Analysis Predicting Changes in Personality Traits From Subjective Age

\begin{tabular}{|c|c|c|c|c|c|}
\hline Variables & Agreeableness & Extraversion & Neuroticism & Conscientiousness & Openness \\
\hline \multicolumn{6}{|l|}{ Mean-level changes } \\
\hline Sex & $-0.11 * * *$ & $-0.03 *$ & $-0.04 * *$ & $-0.03^{*}$ & 0.01 \\
\hline Age & $0.06 * * *$ & $0.05^{* * *}$ & $-0.09 * * *$ & $-0.03 *$ & 0.01 \\
\hline Age squared & $-0.05 * * *$ & $-0.05 * * *$ & 0.02 & $-0.05 * * *$ & $-0.06 * * *$ \\
\hline Educational level & $-0.05 * * *$ & -0.02 & $-0.03 *$ & 0.00 & $0.06 * * *$ \\
\hline Disease burden & -0.00 & -0.02 & $0.06 * * *$ & $-0.06 * * *$ & 0.02 \\
\hline Ethnicity & -0.01 & $-0.05 * * *$ & -0.00 & -0.00 & $-0.03^{*}$ \\
\hline Baseline personality trait & $0.60 * * *$ & $0.68 * * *$ & $0.60 * * *$ & $0.60 * * *$ & $0.67^{* * *}$ \\
\hline Baseline subjective age & $0.04 * *$ & $0.03 *$ & -0.01 & $0.04 * *$ & $0.06^{* * * *}$ \\
\hline Adjusted $R^{2}$ & .43 & .49 & .42 & .39 & .49 \\
\hline \multicolumn{6}{|l|}{ Absolute changes } \\
\hline Sex & $0.09 * * *$ & $0.04 *$ & 0.02 & $0.06 * * *$ & -0.00 \\
\hline Age & $-0.09 * * *$ & -0.03 & $-0.06 * * *$ & $-0.03 *$ & $-0.04^{*}$ \\
\hline Age squared & $0.05 * *$ & 0.01 & $0.03 *$ & 0.03 & $0.07 * * *$ \\
\hline Educational level & $-0.03 *$ & $-0.05^{* *}$ & $-0.04 *$ & $-0.08 * * *$ & $-0.10 * * *$ \\
\hline Disease burden & -0.01 & 0.03 & $0.07 * * *$ & $0.04 *$ & 0.02 \\
\hline Ethnicity & $-0.04 * *$ & $-0.04 *$ & -0.01 & -0.01 & $-0.04 * *$ \\
\hline Baseline subjective age & $-0.05 * *$ & $-0.06 * *$ & $-0.04 *$ & -0.02 & $-0.05^{* *}$ \\
\hline Adjusted $R^{2}$ & .02 & .01 & .01 & .01 & .02 \\
\hline Subjective age changes ${ }^{\mathrm{a}}$ & 0.00 & $-0.03 *$ & 0.02 & $-0.04 *$ & $-0.04 *$ \\
\hline Subjective age changes squared ${ }^{a}$ & $0.05 * *$ & $0.06 * * *$ & 0.02 & $0.05 * *$ & 0.03 \\
\hline Adjusted $R^{2}$ & .02 & .01 & .01 & .01 & .02 \\
\hline
\end{tabular}

Note. $N=3,617$. Coefficients are standardized regression coefficients.

${ }^{a}$ Adjusted for sex, age, age squared, educational level, disease burden, and ethnicity.

$* p<.05$. ** $p<.01$. *** $p<.001$.

the demographic and health covariates. Consistent with our hypothesis, changes in subjective age were positively related to changes in Extraversion, Openness, and Conscientiousness and negatively associated with changes in Neuroticism; although not hypothesized, changes in subjective age were also associated with changes in Agreeableness (see Table 1). The relations were similar without the covariates. To illustrate these continuous effects, we plotted the change in personality for the three subjective aging groups (i.e., slower, stable, accelerated; see Figure 1). These results suggested that feeling increasingly younger or remaining stable was associated with the maintenance of Extraversion, Openness, and Agreeableness, and an increase in Conscientiousness, whereas accelerated subjective aging was associated with a decline in these four traits. Individuals with slower or stable subjective aging decreased more in Neuroticism than those who experienced accelerated aging.

Additional analyses were conducted to examine the relationship between baseline and changes in subjective age and absolute personality changes. The results revealed that baseline subjective age was negatively related to absolute change in Agreeableness $(\beta=-.05, p<.01)$, Extraversion $(\beta=-.06$, $p<.01)$, and Openness $(\beta=-.05, p<.01$; see Table 2$)$. This pattern of results suggests that a younger subjective age at baseline is associated with less absolute change in these three traits. A quadratic relation was found between changes in subjective age and Agreeableness $(\beta=.05, p<.01)$, Extraversion $(\beta=.06, p<.01)$, and Conscientiousness $(\beta=.05, p<.01$; see Table 2). Thus, absolute changes in these traits are larger when individuals feel increasingly younger or older compared to a stable subjective age.

\section{Subjective Age and Indices of Personality Stability}

Across the three measures of stability, we generally found support for our hypothesis that an older subjective age and deviations from the normative trajectory of subjective age would be associated with lower stability.

Rank Order. The Fisher's $z$-test indicated that a younger baseline subjective age was associated with higher rank-order stability than an older baseline subjective age for Extraversion $(z=-2.61, p<.01)$, Openness $(z=-2.00, p<.05)$, and Agreeableness $(z=-2.07, p<.05$; see Table 3$)$. Participants with an average subjective age did not differ from either those with a younger subjective age or those with an older subjective age.

Similar analyses were conducted to test whether changes in subjective age between baseline and follow-up were associated with the rank-order stability of personality. Compared to the stable aging group, the accelerated aging group was less stable in Extraversion $(z=-2.28, p<.05)$ and Openness $(z=-4.47$, 


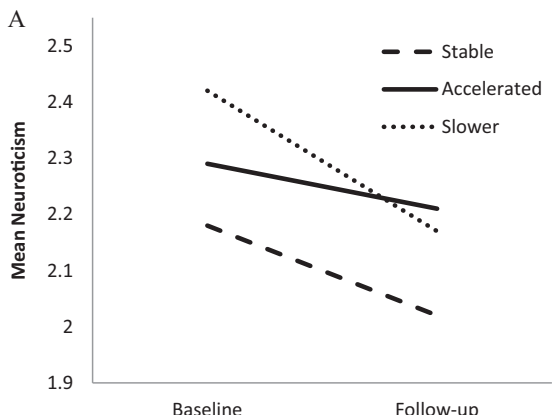

$\mathrm{B}$

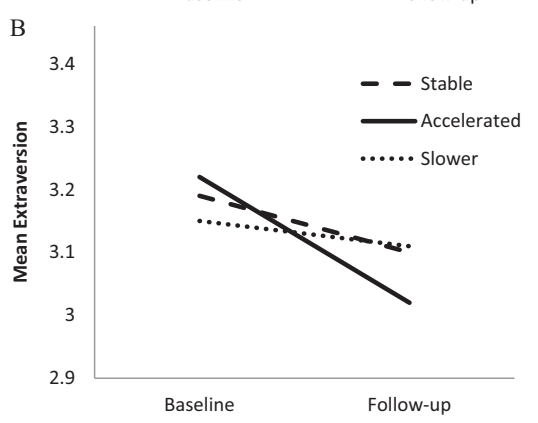

C

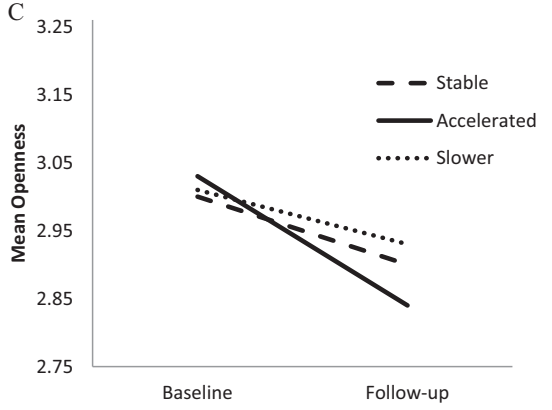

$\mathrm{D}$

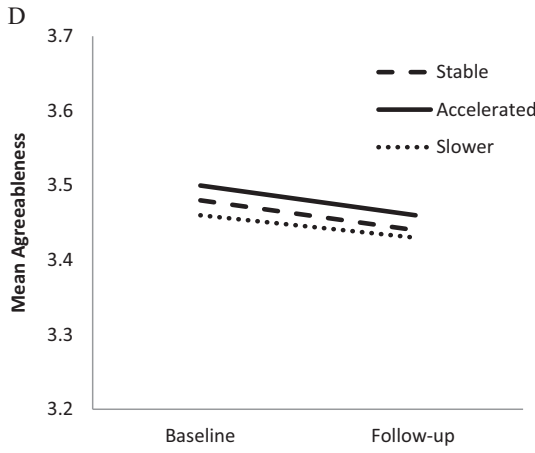

E

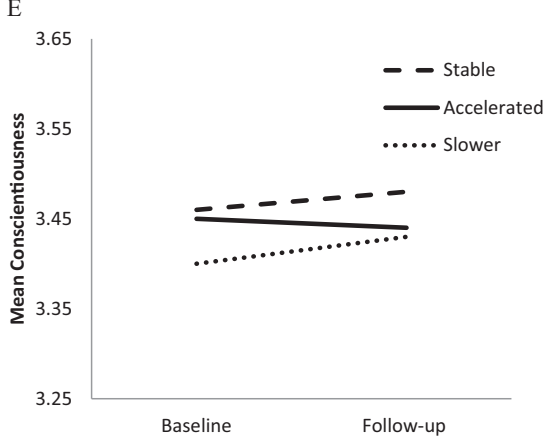

Figure I Changes in Neuroticism (Panel A), Extraversion (Panel B), Openness (Panel C), Agreeableness (Panel D), and Conscientiousness (Panel E) for accelerated, stable, and slower aging groups. $p<.01$; see Table 3 ). The slower aging group was less stable in Agreeableness $(z=-2.68, p<.01)$, Extraversion $(z=-3.44$, $p<.01)$, and Openness $(z=-2.49, p<.05)$ than the stable aging group (Table 3 ). These results suggested that individuals with either accelerated or slower subjective aging were likely to experience non-normative changes in Extraversion, Openness, and Agreeableness, which reduces rank-order stability in these personality domains.

Individual. Regression analysis revealed that a younger subjective age at baseline was associated with higher IS for Extraversion $(\beta=.05, p<.01)$ and higher overall intra-individual stability of personality traits $(\beta=.06, p<.01$; see Table 4$)$. These effects were of similar magnitude to the effects of disease burden, sex, and ethnicity, and were significant even when the covariates were not included in the regression model. Subjective age was also associated with the IS for Agreeableness $(\beta=.07, p<.01)$, Neuroticism $(\beta=.05, p<.01)$, Conscientiousness $(\beta=.05, p<.01)$, and Openness $(\beta=.04, p<.01)$, but these effects were not significant when the covariates were included in the model (see Table 4).

There were also negative quadratic associations between change in subjective age and IS for Extraversion $(\beta=-.07$, $p<.01)$, Conscientiousness $(\beta=-.06, p<.01)$, and IS $\mathrm{S}_{\mathrm{MIDI}}$ ( $\beta=-.07, p<.01)$, either with or without controlling for the demographic and health variables. The effect of subjective age was roughly comparable to that of education and tended to be stronger than disease burden, ethnicity, and sex (Table 4). Although there were also negative quadratic relations between change in subjective age and IS for Agreeableness $(\beta=-.05$, $p<.01)$ and Openness $(\beta=-.04, p=.01)$, these effects were not significant with the inclusion of covariates. These effects indicated that individuals with either slower or accelerated subjective aging over the 10 years were also more likely to experience non-normative personality change, particularly for Extraversion and Conscientiousness.

Profile. Similar analyses were conducted to examine the association between subjective age and intra-individual stability of the configuration of traits, labeled as profile or ipsative stability (Table 4). The results revealed that those who had a younger subjective age at baseline had a more stable profile $(\beta=.09$, $p<.01$ ), with or without controlling for the covariates. The effect of subjective age was roughly similar to that of education, sex, and ethnicity.

Consistent with the previous results, and with or without covariates in the model, a quadratic model was found to represent the relationship between change in subjective age and profile stability $(\beta=-.08, p<.01)$ : Participants who felt either increasingly older or increasingly younger over time had lower profile stability compared to participants who maintained a stable discrepancy between their subjective and chronological age. This association was roughly similar in magnitude to the effect we found for education and sex, and it was larger than what we found for ethnicity (Table 4). 
Table 3 Rank-Order Stability for the Full Sample and by Subjective Age Groups

\begin{tabular}{lccccccc}
\hline Variables & $\begin{array}{c}\text { Total Sample } \\
(N=3,617)\end{array}$ & $\begin{array}{c}\text { Saseline Older } \\
\text { Subjective Age } \\
(n=385)\end{array}$ & $\begin{array}{c}\text { No Discrepancy } \\
\text { at Baseline } \\
(n=319)\end{array}$ & $\begin{array}{c}\text { Baseline Younger } \\
\text { Subjective Age } \\
(n=29 \mid 3)\end{array}$ & $\begin{array}{c}\text { Accelerated } \\
\text { Aging } \\
(n=333)\end{array}$ & $\begin{array}{c}\text { Stable } \\
\text { Aging } \\
(n=2,876)\end{array}$ & $\begin{array}{c}\text { Slower } \\
\text { Aging } \\
(n=408)\end{array}$ \\
\hline Agreeableness & .64 & $.58^{\mathrm{a}}$ & .62 & .65 & .65 & .65 \\
Extraversion & .70 & $.62^{\mathrm{a}}$ & .68 & .70 & $.65^{\mathrm{b}}$ & .72 & $.66^{\mathrm{c}}$ \\
Neuroticism & .64 & .61 & .60 & .64 & .64 & .64 \\
Conscientiousness & .62 & .58 & .65 & .61 & .55 & .62 \\
Openness & .69 & $.64^{\mathrm{a}}$ & .65 & .70 & $.57^{\mathrm{b}}$ & .72 \\
\hline
\end{tabular}

Note. All coefficients are significant at $p<.001$.

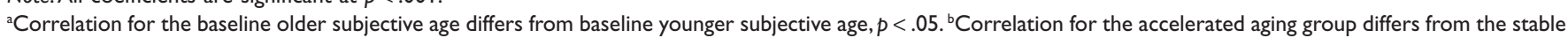
aging group, $p<.05$. $^{c}$ Correlation for the slower aging group differs from the stable aging group, $p<.05$.

Table 4 Summary of Regression Analysis Predicting Intra-Individual Stability of Personality Traits and Profile Consistency From Baseline Subjective Age and Changes in Subjective Age

\begin{tabular}{|c|c|c|c|c|c|c|c|}
\hline Variables & $\mathrm{IS}_{\text {Agreeableness }}$ & $\mathrm{IS}_{\text {Extraversion }}$ & $\mathrm{IS}_{\text {Neuroticism }}$ & $I_{\text {Conscientiousness }}$ & $\mathrm{IS}_{\text {Openness }}$ & $\mid S_{\text {MIDI }}$ & Profile Consistency \\
\hline \multicolumn{8}{|l|}{ Baseline subjective age } \\
\hline Sex & $-0.06 * * *$ & -0.03 & -0.01 & $-0.04 *$ & 0.01 & $-0.05^{* *}$ & $-0.09 * * *$ \\
\hline Age & $0.09 * * *$ & 0.03 & $0.06 * * *$ & 0.02 & $0.03 *$ & $0.08 * * *$ & $0.17 * * *$ \\
\hline Age squared & $-0.06 * * *$ & 0.00 & $-0.05^{* *}$ & $-0.04 *$ & $-0.05^{* *}$ & $-0.07 * * *$ & $-0.06 * * *$ \\
\hline Educational level & $0.05^{* *}$ & $0.07 * * *$ & 0.02 & $0.07 * * *$ & $0.09 * * *$ & $0.10 * * *$ & $0.10 * * *$ \\
\hline Disease burden & 0.01 & $-0.04 *$ & $-0.04 *$ & $-0.05^{* *}$ & $-0.04^{*}$ & $-0.05^{* *}$ & $-0.14^{* * *}$ \\
\hline Ethnicity & $0.05 * *$ & $0.05^{* *}$ & 0.02 & 0.02 & $0.07 * * *$ & $0.07 * * *$ & 0.02 \\
\hline Subjective age & $0.04 *$ & $0.05 * *$ & 0.02 & 0.03 & 0.02 & $0.06 * * *$ & $0.09 * * *$ \\
\hline Adjusted $R^{2}$ & .02 & .01 & .01 & .01 & .02 & .03 & .07 \\
\hline \multicolumn{8}{|l|}{ Changes in subjective age } \\
\hline Sex & $-0.06 * * *$ & -0.03 & -0.01 & $-0.04 * *$ & 0.01 & $-0.05 * *$ & $-0.10 * * *$ \\
\hline Age & $0.09 * * *$ & 0.03 & $0.07^{* * *}$ & 0.02 & $0.04 *$ & $0.09 * * *$ & $0.19 * * *$ \\
\hline Age squared & $-0.06 * * *$ & 0.00 & $-0.05^{* *}$ & $-0.04 *$ & $-0.05^{* *}$ & $-0.07 * * *$ & $-0.07 * * *$ \\
\hline Educational level & $0.05^{* *}$ & $0.06 * * *$ & 0.02 & $0.07^{* * *}$ & $0.09 * * *$ & $0.10 * * *$ & $0.10 * * *$ \\
\hline Disease burden & 0.01 & $-0.04 *$ & $-0.05^{* *}$ & $-0.05^{* *}$ & $-0.04^{*}$ & $-0.05^{* *}$ & $-0.15^{* * *}$ \\
\hline Ethnicity & $0.04^{* *}$ & $0.05^{* *}$ & 0.02 & 0.02 & $0.07 * * *$ & $0.06 * * *$ & 0.02 \\
\hline Subjective age changes & -0.01 & -0.00 & 0.00 & 0.03 & 0.02 & 0.01 & 0.03 \\
\hline Subjective age changes squared & $-0.04 *$ & $-0.07 * * *$ & -0.02 & $-0.06 * * *$ & -0.03 & $-0.07 * * *$ & $-0.08 * * *$ \\
\hline Adjusted $R^{2}$ & .02 & .01 & .01 & .01 & .02 & .03 & .07 \\
\hline
\end{tabular}

Note. $N=3,6 I 7$. IS = intra-individual stability. IS was computed as I $-\left[\left(z_{1}-z_{2}\right)^{2} / 2\right]$. IS MIDI is the mean IS of the five factors. Profile consistency was computed using the double-entry intra-class correlation across the five personality traits. Coefficients are standardized regression coefficients.

$*_{p}<.05$. ${ }^{* *} p<.01$. ***p $<.001$

\section{DISCUSSION}

This study examined the longitudinal dynamics between subjective age and personality traits over approximately 10 years. Independent of chronological age, subjective age and accelerated subjective aging were associated with theoretically meaningful changes in characteristic ways of feeling, thinking, and behaving. These findings suggest that the subjective experience of aging, which reflects both biological and psychosocial aging, is one alternative indicator of developmental time that contributes significantly to personality development in adulthood.

\section{Subjective Age and Personality Development}

In adulthood, personality traits tend to be relatively stable and follow normative age-related trajectories over time (Lucas \&
Donnellan, 2011; Roberts \& DelVecchio, 2000; Terracciano et al., 2006, 2010); however, non-normative changes are particularly informative in identifying factors that contribute to personality development. In the present research, we found that a younger subjective age and maintaining a stable subjective age - which are normative phenomena in adulthood and old age (Gana et al., 2004; Rubin \& Berntsen, 2006) - are related to normative changes in personality traits. Conversely, non-normative trajectories in personality were observed among participants who felt older than their chronological age at baseline and among those who deviated from the normative trajectory of subjective age over time.

We hypothesized that subjective age at baseline and changes in subjective age over the follow-up period would be associated with changes in Extraversion and Openness. 
Although Extraversion was not found to vary according to baseline subjective age, participants who experienced accelerated subjective aging between baseline and follow-up had the steepest declines in Extraversion and Openness, whereas participants with average or slower subjective aging showed the more typical declines in these traits. That is, those who felt relatively older over time declined more in the tendency to be energetic, enthusiastic, sociable, and excitement seeking (Extraversion) and in their propensity for intellectual curiosity and preference for variety, novel ideas, and new experiences (Openness) over time than those with a younger subjective age.

These changes may be mediated by both social and physiological mechanisms. For example, when experimentally induced to feel older, individuals tend to endorse attitudes and opinions stereotypically associated with older people (Eibach et al., 2010). With regard to personality, older adults are stereotypically perceived as particularly introverted and closed-minded (Chan et al., 2012). As such, individuals who feel subjectively older may come to identify with the traits that are commonly thought to characterize older adults. Physiologically, older subjective age reflects, in part, declines in physical and cognitive health (Demakakos et al., 2007; Keyes \& Westerhof, 2012; Kotter-Grühn et al., 2009; Stephan et al., in press), and individuals may feel subjectively older when their energy levels start to decline. With loss of energy, the exuberance of Extraversion may be hit particularly hard. Likewise, the cognitive decline associated with subjective age may contribute to a decline in the interest in learning new things, being creative, and being flexible. Of note, the effect of subjective age on changes in personality persisted even after accounting for comorbid disease burden.

We found partial support for our hypothesis for an effect of subjective age on changes in Neuroticism: Although baseline subjective age was unrelated to changes in Neuroticism over time, changes in subjective age over the follow-up were related to changes in Neuroticism. Those who maintained a stable subjective age and those who felt increasingly younger declined in Neuroticism, whereas those who experienced accelerated subjective aging maintained a stable propensity to experience negative emotions. This pattern may be indicative of increased disease burden and conditions, cognitive decline, and emerging depressive symptoms, which impact both subjective age (Keyes \& Westerhof, 2012; KleinspehnAmmerlahn et al., 2008; Kotter-Grühn et al., 2009) and Neuroticism (Löckenhoff, Terracciano, \& Costa, 2009).

Feeling younger at baseline and feeling subjectively younger over time were also associated with an increase in Conscientiousness and less decline in Agreeableness. The self-regulation processes leading to an increasing younger subjective age over time are likely to be reflected in higher Conscientiousness. A younger subjective age is an indicator of self-regulation processes and adaptive capacities (Infurna et al., 2010; Kleinspehn-Ammerlahn et al., 2008; KotterGrühn et al., 2009) and is related to health behavior such as physical activity (Stephan et al., in press). Individuals who feel subjectively younger may be healthier and thus have a greater ability to behave in a more conscientious manner over time than individuals with declining health who feel subjectively older. Furthermore, a younger subjective age relies in part on favorable social interactions (Stephan et al., 2013). Therefore, individuals who feel younger may be more likely to be socially engaged and to search for and benefit from positive social interactions, leading to maintenance of prosocial behavior (i.e., Agreeableness), compared to those with an older subjective age. These results suggest that a younger subjective age is associated with more adaptive trajectories than an older subjective age.

Taken together, our results reveal that changes in the discrepancy between subjective and chronological age are associated with meaningful mean-level personality changes. The decrease in both Extraversion and Openness observed among those who felt older between baseline and follow-up corresponded roughly to a one-third standard deviation decline over a 10 -year period. The decrease in Neuroticism in individuals who felt increasingly younger corresponded to a one-third standard deviation decrease. These changes are larger than the age-related change generally observed during adulthood (Terracciano et al., 2005) and are similar in magnitude to the personality changes associated with extreme events (Löckenhoff, Terracciano, Patriciu, Eaton, \& Costa, 2009), military training (Jackson et al., 2012), and weight gain (Sutin, Costa et al., 2013).

In addition to mean-level change, subjective age and changes in subjective age were associated with a number of indices of personality stability. In general, a younger baseline subjective age was associated with higher stability and greater profile consistency, independent of other recognized predictors, such as chronological age, education, disease burden, and ethnicity (Lucas \& Donnellan, 2011; Löckenhoff, Terracciano, \& Costa, 2009; Terracciano et al., 2010). The strength of the associations between subjective age and the indices of stability was comparable to the effects observed for some of the demographic variables and for disease burden.

Consistent with conceptualizations of a younger subjective age as a self-protective strategy (Weiss \& Freund, 2012; Weiss \& Lang, 2012), it is likely that feeling younger than one's chronological age may help preserve stability. Individuals with a youthful subjective age possess characteristics that allow them to successfully deal with age-related changes (Stephan et al., 2011), resulting in better physical health (Kotter-Grühn et al., 2009), well-being (Stephan et al., 2011), cognitive functioning (Stephan et al., in press), and efficient protection from negative aging stereotypes (Eibach et al., 2010; Mock \& Eibach, 2011). As such, individuals who feel younger than their age at baseline may be better equipped to buffer physical, cognitive, and social changes that challenge personality stability, whereas feeling older may reflect a vulnerability to aging stereotypes or deteriorating health that may challenge stability. However, there was an inverted-U-shaped pattern to the relation between changes in subjective age and stability: 
Accelerated aging and slower aging were both associated with lower intra-individual and profile stabilities compared to average aging. Complementarily, a U-shaped pattern was found for absolute personality change, which suggests that both accelerated aging and slower aging are associated with higher total amount of personality change. It thus appears that lower personality stability is not restricted to accelerated aging, but may result from any deviation from normative trajectories of subjective age. It seems reasonable to speculate that the life experiences or biomedical changes that bring about changes in subjective age (in either direction) are likely to be associated with personality changes away from the normative trajectories, which would reduce stability.

\section{STRENGTHS, LIMITATIONS, AND FUTURE DIRECTIONS}

The results of this research suggest that the subjective experience of aging is a meaningful indicator of developmental time associated with stability and change in personality. Previous research has focused primarily on the effect of chronological age (Lucas \& Donnellan, 2011; Specht et al., 2011), ethnicity and education (Löckenhoff et al., 2008), life experiences and normative life events (Löckenhoff, Terracciano, Patriciu et al., 2009; Roberts, Wood, \& Smith, 2005), physical health (Sutin, Zonderman et al., 2013), and cognitive status (Robins Wahlin \& Byrne, 2011) on personality development across adulthood and old age. The present research adds subjective age to this growing list.

The present research had a number of strengths, including a large sample, a 10-year time frame, two assessments of both personality and subjective age that allowed us to examine the reciprocal relations between the two, and multiple indices of stability. The current study also had several limitations that should be considered when interpreting the results. Although the contribution of subjective age on personality change was almost comparable to other known predictors, the effect sizes were relatively small. The generalizability of our findings is limited to some extent by the positive selection of the longitudinal participants in the MIDUS (Graham \& Lachman, 2012; Turiano et al., 2012). More precisely, the contribution of changes in subjective age observed in the present study may underestimate the true contribution given that participants who dropped out were feeling older and thus could have experienced more personality changes. The two-wave design of the MIDUS provides limited opportunity to assess change over time. A multiwave design could provide more reliable information on the reciprocal dynamic between both constructs, would allow for testing whether these changes are temporary or whether they persist, and would be a stronger test of nonlinear changes over time. Although the measure of personality has acceptable psychometric properties, it assessed only the five broad dimensions. Future research would benefit from adopting a facetlevel analysis to provide a more in-depth picture of the relation between changes in subjective age and changes in personality.
The findings of the present study are based on an American sample, and other studies have reported cross-cultural differences in the discrepancy between chronological and subjective ages (Westerhof et al., 2003): Americans tend to have a more youthful subjective age than their European counterparts because of the strong youth-centeredness of American culture (Westerhof et al., 2003). Furthermore, feeling younger has stronger implications for Americans than Europeans, such as for subjective well-being (Westerhof \& Barrett, 2005). Therefore, future studies are needed to test whether our findings generalize to samples from other countries, and whether culture influences the association between changes in subjective age and changes in personality traits. Most participants were between ages 35 and 60 at baseline. Therefore, the present results are mostly informative about subjective age and personality development across middle adulthood. Additional studies with older and younger adults are needed to examine the relationship between subjective age and personality development across the full life span. Longitudinal studies are also needed to examine the mediating role of cognitive, physical, and mental health changes in this relation.

Despite these limitations, this study provides new evidence for factors associated with personality development. Although chronological age is typically used as a key indicator of personality change, alternative ways of measuring developmental time, such as the assessment of the subjective experience of age, provide valuable insights about the mechanisms involved in personality development across the adult life span.

\section{References}

Agrigoroaei, S., \& Lachman, M. E. (2011). Cognitive functioning in midlife and old age: Combined effects of psychosocial and behavioral factors. Journals of Gerontology, Series B: Psychological Sciences and Social Sciences, 66, 130-140.

Ardelt, M. (2000). Still stable after all these years? Personality stability theory revisited. Social Psychology Quarterly, 63, 392405.

Asendorpf, J. B. (1992). Beyond stability: Predicting inter-individual differences in intra-individual change. European Journal of Personality, 6, 103-117.

Barrett, A. E. (2003). Socioeconomic status and age identity: The role of dimensions of health in the subjective construction of age. Journals of Gerontology, Series B: Psychological Sciences and Social Sciences, 58, S101-S109.

Brim, O. G., Ryff, C. D., \& Kessler, R. C. (Eds.). (2004). How healthy are we? A national study of well-being at midlife. Chicago: University of Chicago Press.

Canada, B., Stephan, Y., Caudroit, J., \& Jaconelli, A. (2013). Personality and subjective age among older adults: The mediating role of age-group identification. Aging and Mental Health, 17, 10371043.

Chan, W., McCrae, R. R., De Fruyt, F., Jussim, L., Löckenhoff, C. E., De Bolle, M., ... Terracciano, A. (2012). Stereotypes of age 
differences in personality traits: Universal and accurate? Journal of Personality and Social Psychology, 103, 1050-1066.

De Fruyt, F. D., Bartels, M., Van Leeuwen, K. G., De Clercq, B., Decuyper, M., \& Mervielde, I. (2006). Five types of personality continuity in childhood and adolescence. Journal of Personality and Social Psychology, 91, 538-552.

Demakakos, P., Gjonca, E., \& Nazroo, J. (2007). Age identity, age perceptions, and health: Evidence from the English Longitudinal Study of Ageing. Annals of the New York Academy of Sciences, 1114, 279-287.

Donnellan, M. B., \& Lucas, R. E. (2008). Age differences in the Big Five across the life span: Evidence from two national samples. Psychology and Aging, 23, 558-566.

Donnellan, M. B., \& Robins, R. W. (2009). The development of personality across the life span. In P. J. Corr \& G. Matthews (Eds.), Cambridge handbook of personality (pp. 191-204). New York: Cambridge University Press.

Eibach, R. P., Mock, S. E., \& Courtney, E. A. (2010). Having a "senior moment": Induced aging phenomenology, subjective age, and susceptibility to ageist stereotypes. Journal of Experimental Social Psychology, 46, 643-649.

Galambos, N. L., Turner, P. K., \& Tilton-Weaver, L. C. (2005). Chronological and subjective age in emerging adulthood: The crossover effect. Journal of Adolescent Research, 20, 538 -556.

Gana, K., Alaphilippe, D., \& Bailly, N. (2004). Positive illusions and mental and physical health in later life. Aging and Mental Health, 8, 58-64.

Graham, E. K., \& Lachman, M. E. (2012). Personality stability is associated with better cognitive performance in adulthood: Are the stable more able? Journals of Gerontology, Series B: Psychological Sciences and Social Sciences, 67, 545-554.

Helson, R., Kwan, V. S., John, O. P., \& Jones, C. J. (2002). The growing evidence for personality change in adulthood: Findings from research with personality inventories. Journal of Research in Personality, 36, 287-306.

Hubley, A. M., \& Hultsch, D. F. (1994). The relationship of personality trait variables to subjective age identity in older adults. Research on Aging, 16, 415-439.

Hubley, A. M., \& Hultsch, D. F. (1996). Subjective age and traits. Research on Aging, 18, 494-496.

Hubley, A. M., \& Russell, L. B. (2009). Prediction of subjective age, desired age, and age satisfaction in older adults: Do some health dimensions contribute more than others? International Journal of Behavioral Development, 33, 12-21.

Infurna, F. J., Gerstorf, D., Robertson, S., Berg, S., \& Zarit, S. H. (2010). The nature and cross-domain correlates of subjective age in the oldest-old: Evidence from the OCTO study. Psychology and Aging, 25, 470-476.

Jackson, J. J., Thoemmes, F., Jonkmann, K., Lüdtke, O., \& Trautwein, U. (2012). Military training and personality trait development: Does the military make the man or does the man make the military? Psychological Science, 23, 270-277.

Kastenbaum, R., Derbin, V., Sabatini, P., \& Artt, S. (1972). "The ages of me": Toward personal and interpersonal definitions of functional aging. Aging and Human Development, 3, 197-211.
Keyes, C. L. M., \& Westerhof, G. J. (2012). Chronological and subjective age differences in flourishing mental health and major depressive episode. Aging and Mental Health, 16(1), 67-74.

Kleinspehn-Ammerlahn, A., Kotter-Grühn, D., \& Smith, J. (2008). Self-perceptions of aging: Do subjective age and satisfaction with aging change during old age? Journals of Gerontology, Series B: Psychological Sciences and Social Sciences, 63, 377-385.

Knoll, N., Rieckmann, N., Scholz, U., \& Schwarzer, R. (2004). Predictors of subjective age before and after cataract surgery: Conscientiousness makes a difference. Psychology and Aging, 19, 676-688.

Kotter-Grühn, D., Kleinspehn-Ammerlahn, A., Gerstorf, D., \& Smith, J. (2009). Self-perceptions of aging predict mortality and change with approaching death: 16-year longitudinal results from the Berlin Aging Study. Psychology and Aging, 24, 654-667.

Lachman, M. E., \& Weaver S. L. (1997). The Midlife Development Inventory (MIDI) Personality Scales: Scale construction and scoring. Technical report. Retrieved from http://www.brandeis .edu/departments/psych/lachman/pdfs/midi-personality-scales .pdf

Löckenhoff, C. E., Terracciano, A., Bienvenu, O. J., Patriciu, N. S., Nestadt, G., McCrae, R. R., . . Costa Jr. , P. T., (2008). Ethnicity, education, and the temporal stability of personality traits in the East Baltimore Epidemiologic Catchment Area Study. Journal of Research in Personality, 42, 577-598.

Löckenhoff, C. E., Terracciano, A., \& Costa, P. T., Jr. (2009). Fivefactor model personality traits and the retirement transition: Longitudinal and cross-sectional associations. Psychology and Aging, 24, 722-728.

Löckenhoff, C. E., Terracciano, A., Patriciu, N. S., Eaton, W. W., \& Costa, P. T., Jr. (2009).Self-reported extremely adverse life events and longitudinal changes in five-factor model personality traits in an urban sample. Journal of Traumatic Stress, 22, 53-59.

Lucas, R. E., \& Donnellan, M. B. (2009). Age differences in personality: Evidence from a nationally representative Australian sample. Developmental Psychology, 45, 1353-1363.

Lucas, R. E., \& Donnellan, M. B. (2011). Personality development across the life span: Longitudinal analyses with a national sample from Germany. Journal of Personality and Social Psychology, 101, 847-861.

Lüdtke, O., Roberts, B. W., Trautwein, U., \& Nagy, G. (2011). A random walk down the university avenue: Life paths, life events, and personality trait change at the transition to university life. Journal of Personality and Social Psychology, 101, 620-637.

MacDonald, S. W. S., DeCarlo, C. A., \& Dixon, R. A. (2011). Linking biological and cognitive aging: Towards improving characterizations of developmental time. Journals of Gerontology, Series B: Psychological Sciences and Social Sciences, 66, 59-70.

McCrae, R. R. (2008). A note on some measures of profile agreement. Journal of Personality Assessment, 90, 105-109.

McCrae, R. R., Terracciano, A., \& Members of the Personality Profiles of Cultures Project. (2005). Universal features of personality traits from the observer's perspective: Data from 50 cultures. Journal of Personality and Social Psychology, 88, 547-561. 
Mock, S. E., \& Eibach, R. P. (2011). Aging attitudes moderate the effect of subjective age on psychological well-being: Evidence from a 10-year longitudinal study. Psychology and Aging, 26, 979-986.

Montepare, J. M. (2009). Subjective age: Toward a guiding lifespan framework. International Journal of Behavioral Development, 33, $42-46$.

Mroczek, D. K., \& Spiro, A. (2003). Modeling intraindividual change in personality traits: Findings from the Normative Aging Study. Journals of Gerontology, Series B: Psychological Sciences and Social Sciences, 58, 153-165.

Pullmann, H., Raudsepp, L., \& Allik, J. (2006). Stability and change in adolescents' personality: A longitudinal study. European Journal of Personality, 20, 447-459.

Radler, B. T., \& Ryff, C. D. (2010). Who participates? Accounting for longitudinal retention in the MIDUS National Study of Health and Well-Being. Journal of Aging and Health, 22, 307-331.

Roberts, B. W., Caspi, A., \& Moffitt, T. (2001). The kids are alright: Growth and stability in personality development from adolescence to adulthood. Journal of Personality and Social Psychology, 81, 670-683.

Roberts, B. W., \& DelVecchio, W. F. (2000). The rank-order consistency of personality traits from childhood to old age: A quantitative review of longitudinal studies. Psychological Bulletin, 126, 3-25.

Roberts, B. W., Walton, K. E., \& Viechtbauer, W. (2006). Patterns of mean-level change in personality traits across the life course: A meta-analysis of longitudinal studies. Psychological Bulletin, 132, 1-25.

Roberts, B. W., Wood, D., \& Smith, J. L. (2005). Evaluating five factor theory and social investment perspectives on personality trait development. Journal of Research in Personality, 39, 166-184.

Robins Wahlin, T. B., \& Byrne, G. J. (2011). Personality changes in Alzheimer's disease: A systematic review. International Journal of Geriatric Psychiatry, 26, 1019-1029.

Rubin, D. C., \& Berntsen, D. (2006). People over forty feel $20 \%$ younger than their age: Subjective age across the life span. Psychonomic Bulletin and Review, 13, 776-780.

Small, B. J., Hertzog, C., Hultsch, D. F., \& Dixon, R. A. (2003). Stability and change in adult personality over 6 years: Findings from the Victoria Longitudinal Study. Journals of Gerontology, Series B: Psychological Sciences and Social Sciences, 58, 166176.

Soto, C. J., John, O. P., Gosling, S. D., \& Potter, J. (2011). Age differences in personality traits from 10 to 65: Big-Five domains and facets in a large cross-sectional sample. Journal of Personality and Social Psychology, 100, 330-348.

Specht, J., Egloff, B., \& Schmukle, S. C. (2011). Stability and change of personality across the life course: The impact of age and major life events on mean-level and rank-order stability of the Big Five. Journal of Personality and Social Psychology, 101, 862-882.

Stephan, Y., Caudroit, J., Jaconelli, A., \& Terracciano, A. (in press). Subjective age and cognitive functioning: A 10-year prospective study. American Journal of Geriatric Psychiatry. doi:10.1016/ j.jagp.2013.03.007
Stephan, Y., Caudroit, J., \& Chalabaev, A. (2011). Subjective health and memory self-efficacy as mediators in the relation between subjective age and life satisfaction among older adults. Aging and Mental Health, 15, 428-437.

Stephan, Y., Chalabaev, A., Kotter-Grühn, D., \& Jaconelli, A. (2013). "Feeling younger, being stronger": An experimental study of subjective age and physical functioning among older adults. Journals of Gerontology Series B: Psychological Sciences and Social Sciences, 68, 1-7.

Stephan, Y., Demulier, V., \& Terracciano, A. (2012). Personality, self-rated health and subjective age in a life-span sample: The moderating role of chronological age. Psychology and Aging, 27, $875-880$.

Sutin, A. R., Costa Jr., P. T., Chan, W., Milaneschi, Y., Eaton, W. W., Zonderman, A. B., . . . Terracciano, A. (2013). I know not to, but I can't help it: Weight gain and changes in impulsivity-related personality traits. Psychological Science, 24, 1323-1328.

Sutin, A. R., Zonderman, A. B., Ferrucci, L., \& Terracciano, A. (2013). Personality traits and chronic disease: Implications for adult personality development. Journals of Gerontology, Series B: Psychological Sciences and Social Sciences, 68, 912-920.

Takahashi, Y., Edmonds, G. E., Jackson, J. J., \& Roberts, B. W. (2013). Longitudinal correlated changes in conscientiousness, preventative health-related behaviors, and self-perceived physical health. Journal of Personality, 81, 417-427.

Terracciano, A., McCrae, R. R., Brant, L. J., \& Costa, P. T., Jr. (2005). Hierarchical linear modeling analyses of the NEO-PI-R scale in the Baltimore Longitudinal Study of Aging. Psychology and Aging, 20, 493-506.

Terracciano, A., McCrae, R. R., \& Costa, P. T., Jr. (2006). Longitudinal trajectories in Guilford-Zimmerman Temperament Survey data: Results from the Baltimore Longitudinal Study of Aging. Journals of Gerontology, Series B: Psychological Sciences and Social Sciences, 61, 108-116.

Terracciano, A., McCrae, R. R., \& Costa, P. T., Jr. (2010). Intraindividual change in personality stability and age. Journal of Research in Personality, 44, 31-37.

Trzesniewski, K. H., Donnellan, M. B., \& Robins, R. W. (2003). Stability of self-esteem across the lifespan. Journal of Personality and Social Psychology, 84, 205-220.

Turiano, N. A., Pitzer, L., Armour, C., Karlamangla, A., Ryff, C. D., \& Mroczek, D. K. (2012). Personality trait level and change as predictors of health outcomes: Findings from a national study of Americans (MIDUS). Journals of Gerontology, Series B: Psychological Sciences and Social Sciences, 67, 4-12.

Uotinen, V., Rantanen, T., \& Suutama, T. (2005). Perceived age as a predictor of old age mortality: A 13-year prospective study. Age and Ageing, 34, 368-372.

Uotinen, V., Rantanen, T., Suutama, T., \& Ruoppila, I. (2006). Change in subjective age among older people over an eight-year followup: "Getting older and feeling younger?" Experimental Aging Research, 32, 381-393.

Weiss, D., \& Freund, A. M. (2012). Still young at heart: Negative age-related information motivates distancing from same-aged people. Psychology and Aging, 27, 173-180. 
Weiss, D., \& Lang, F. R. (2012). “They” are old but "I" feel younger: Age-group dissociation as a self-protective strategy in old age. Psychology and Aging, 27, 153-163.

Westerhof, G. J., \& Barrett, A. E. (2005). Age identity and subjective well-being: A comparison of the United States and Germany. Journals of Gerontology, Series B: Psychological Sciences and Social Sciences, 60, S129-S136.
Westerhof, G. J., Barrett, A., \& Steverink, N. (2003). Forever young: A comparison of age identities in the United States and Germany. Research on Aging, 25, 366-383.

Wood, D., \& Roberts, B. W. (2006). The effect of age and role information on expectations for Big Five personality traits. Personality and Social Psychology Bulletin, 32, 1482-1496. 